PR-22

\title{
SYNTHESIS OF A NEW CLASS OF THIAZOLYL MORPHOLINES / THIOMORPHOLINES AND EVALUATION AS ANTIMICROBIALS
}

\author{
G. Sravya, ${ }^{1}$ N. Bakthavatchala Reddy, ${ }^{1}$ and Grigory V Zyryanov ${ }^{1,2}$ \\ ${ }^{1}$ Chemical Engineering Institute, Ural Federal University, Yekaterinburg 620002, Russia. \\ ${ }^{2}$ I. Ya. Postovsky Institute of Organic Synthesis, Ural Division of Russian Academy of Sciences, \\ 22 S. Kovalevskoy St., 620219, Yekaterinburg, Russian Federation. \\ Corresponding author, E-mail: sravyasvu@gmail.com
}

\begin{abstract}
Thiazoles and their derivatives exhibit antibacterial, antifungal and antiinflammatoryactivities. The Hantzsch reaction of $\alpha$-halocarbonyl compounds with thioureas or thioamides is the most commonly used method for the synthesis of thiazoles. They are known to have antimicrobial, anticancer, anti-inflammatory and anti-HIV properties. Morpholine is a heterocyclic organic compound, many of morpholine derivatives have very good biological activity in different therapeutic area such as antibacterial, antiviral, anticancer, antimicrobial, antidiabetic, anti-inflammatory, antimalarial, antifungal etc. Moreover, thiomorpholines analogs are associated with a variety of pharmacological activities including anti mycobacterial, antibacterial, analgesic and anti-inflammatory. In continuation of our studies and also to establish structure-activity relationship of the compounds, the present work synthesis and antimicrobial activity of sulfonyl methyl linked mono and bis-heterocycles having different heterocyclic moieties has been taken up.
\end{abstract}

